\title{
DIDÁCTICAS Y HORIZONTES DE SENTIDO DEL DEPARTAMENTO DE HUMANIDADES EN LA FACULTAD DE DERECHO DE LA UNIVERSIDAD SANTO TOMÁS DE BUCARAMANGA [USTA]
}

Marco Emilio Bautista Buitrago ${ }^{2}$

Dénix Alberto Rodríguez Torres ${ }^{3}$

Alfonso Rodríguez Balaguera ${ }^{4}$

Universidad Santo Tomás - Bucaramanga

\section{Resumen}

Una tarea ineludible del maestro de hoy, es la de constituirse en investigador de su propia práctica. Por lo tanto, el estudio interroga: ¿Dentro de las prácticas pedagógicas aplicadas por el Departamento de Humanidades, qué didácticas generan horizontes de sentido en los estudiantes de Derecho de la Universidad Santo Tomás, seccional Bucaramanga? Dicha pregunta conlleva a una investigación con enfoque cualitativo que, apoyada en la observación etnográfica y en la entrevista profunda, genera información que se canaliza en la metodología de la teoría fundamentada propuesta por Barney Glasser y Anselm Strauss (1967). Además, recaba en el campo de la pedagogía, en torno a las didácticas aplicadas y en el campo filosófico (hermenéutico) en torno a los horizontes de sentido, indagación que define un importante marco teórico que a su vez se contrasta con los datos obtenidos.

La investigación, halla serias divergencias conceptuales en torno al ser y hacer del departamento en sus prácticas pedagógicas desde las categorías indagadas, a la vez que plantea nuevos interrogantes y retos para la seccional, las facultades y departamentos, en dirección a constituir líneas de investigación transdisciplinares, para unificar criterios

\section{Palabras clave:}

Didáctica, Horizonte de sentido, Prácticas pedagógicas.

\section{DIDACTICS AND AIMS IN HUMANITIES EDUCATION SECTION AT LAW FACULTY OF ST. TOMAS AQUINAS UNIVERSITY BUGARAMANGA}

\section{Alistract:}

An unavoidable today educator's duty is to become a researcher of his/her own educational field. So, the study requests: Among the pedagogical practices applied by the

1 El presente artículo de investigación es producto de la investigación "Didácticas y horizontes de sentido del Departamento de Humanidades en la Facultad de Derecho de la Universidad Santo Tomás de Bucaramanga (USTA)", de los autores en el marco de la Maestría en Educación de la Vicerrectoría de la Universidad Abierta y a Distancia (VUAD). Bogotá.

2 Licenciado en Filosofía y Teología de la Universidad Javeriana. Diplomado en Ambientes Virtuales. Universidad Santo Tomás, Bucaramanga. Mg. en Educación (Vuad) 2010. emilbat@hotmail.com

3 Licenciado en Filosofía, Universidad Minuto de Dios; Estudios de Teología en la Universidad Javeriana; Diplomado en Ambientes Virtuales, Universidad Santo Tomás, Bucaramanga. Mg. En Educación (Vuad), 2010. denixvirtual@gmail.com

4 Licenciado en filosofía de la Universidad Javeriana de Bogotá. Especialista en docencia universitaria de la USTA. Diplomado en ambientes virtuales de la USTA. Magíster en educación Vuad 2010. arodriguezbalaguera@hotmail.com 
humanities educational section, which didactics can create belonging sense in law faculty of St. Tomas Aquinas University Bucaramanga? Such question leads to a research with qualitative approach that being supported to ethnographic observation and the deep interview creates canalized information in methodology of the background theory proposed by Barney Glasser and Anselm Strauss (1967). It also deepens in pedagogy field, around applied didactics and philosophy field (Hermeneutics) related to goals; investigation that defines a remarkable frame study even it contrasts with the obtained data.

\section{Key words:}

Didactics, aims, pedagogic practices.

\section{Introducción}

Para la Universidad Santo Tomás, seccional Bucaramanga, el Departamento de Humanidades como unidad académica, centra su labor formativa en la persona del estudiante, de ahí que la universidad se proponga “(...) Promover la formación integral de las personas, en el campo de la Educación Superior, mediante acciones y procesos de enseñanza-aprendizaje, investigación y proyección social, para que respondan de manera ética, creativa y crítica a las exigencias de la vida humana" (PEI, Misión USTA. Estatuto Orgánico de 2002, Art. 7). En este empeño la institución se actualiza y asume los cambios sociales como desafíos para la formación personal y profesional de los jóvenes del oriente colombiano; en igual medida los docentes se cualifican de manera permanente, revisan sus prácticas pedagógicas y buscan alternativas que permitan ofrecer una educación de calidad.

El Departamento de Humanidades constituye en el contexto de la USTA y dentro de la inspiración del Estudio General el ámbito privilegiado de la articulación filosófico-teológica con las diversas disciplinas particulares. "Su actuar propende por la formación teórica y práctica que de una manera armónica dentro de un proceso formativo y complejo desarrolla la cultura científica y la cultura humanística" (USTA, PEI, 2004: 4.3).

Desde los anteriores presupuestos, la investigación busca identificar dentro de las prácticas pedagógicas aplicadas por el Departamento de Humanidades, ¿Qué didácticas generan horizontes de sentido en los estudiantes de Derecho de la Universidad Santo Tomás de Bucaramanga? El problema suscita dos objetivos: por una parte reconocer las didácticas que el Departamento aplica en sus prácticas pedagógicas a estudiantes de la Facultad de Derecho, por otra, definir los horizontes de sentido que generan dichas didácticas en los estudiantes de la facultad en mención.

En el desarrollo de los objetivos, se hace necesaria una revisión documental sobre didácticas y horizontes de sentido en el contexto humanístico de la educación superior, lo que constituye un avance en la consolidación del marco teórico.

Para el logro de estos objetivos se hizo necesario aplicar instrumentos de recolección de información como la observación y la encuesta, apoyados estos por la metodología de la Teoría Fundamentada (Grounded Theory) que, a su vez, orienta la información a la conformación de categorías que son objeto de análisis en el proceso de triangulación.

Luego de este proceso, se abre paso a la determinación de los hallazgos, conclusiones, y recomendaciones.

\section{Aproximaciones Teóricas}

En cumplimiento de su deber y misión, la Universidad Santo Tomás de Bucaraman- 
ga cumple 36 años en la formación de profesionales en el oriente colombiano. De acuerdo a este recorrido histórico, y dados los desafíos de la Educación Superior en el contexto actual, merece revisar de manera particular las prácticas pedagógicas del Departamento de humanidades a fin de hallar las didácticas que allí se aplican en relación a horizontes de sentido. Para ello es pertinente indagar y confrontar con estudios previos, afines al fenómeno en estudio, en aras de la calidad educativa, revisar principios institucionales, planes curriculares y lineamientos para cada Facultad o Departamento; actualizar, con esto, la razón de la formación humana y profesional del educando.

\section{Estudios en torno a Didácticas y Horizontes de Sentido}

Francisco Jarabo Friedrich (2003) del Departamento de Ingeniería Química y Tecnología de la Universidad de Laguna de Salamanca (España), desarrolló un estudio titulado: Investigación en recursos didácticos: Las tecnologías de la información y las comunicaciones. Manifiestó allí un problema humano y social propio del siglo XXI. Anotó que la aplicación de las didácticas se encamina más al uso de las tecnologías de la información y la comunicación (TIC) que a la formación integral humana. Manifiestó que: "existen muchas incertidumbres frente a dichas estructuras generadas y es evidente que la sociedad busca generar conocimiento a partir de la información, por consiguiente es necesario 'aprender a saber' " (Jarabo 2003: 6).

Celso Rivas Balboa (1999) en relación a horizontes de sentido destaca en su texto Investigaciones sobre el sentido, el valor que han dejado los aportes de Víctor Frank, El hombre en busca de sentido (1959). Dice Rivas: "la pregunta por el sentido es la clave de solución dentro de una terapia cognitiva, en situaciones exis- tencialmente relevantes" (Rivas, 1999: 159). Por otra parte, intenta recuperar el sentido de la cotidianidad, desde los adelantos de Lévi-Strauss (1982) sobre la ampliación del horizonte de sentido, y Heinrich Beck (1976, 1979, 1993), formulan la pregunta por el sentido o propósito de la vida." (Rivas, 1999: 166).

Litwit Edith (1997), de la Universidad de Buenos Aires (Argentina) llevó a cabo una investigación titulada: Las configuraciones didácticas, trabajo publicado por la editorial Paidós en 1997. Describe algunas formas de hacer docencia por parte de profesores universitarios en una Facultad específica: la Facultad de Filosofía y Letras, quien pretende reconocer un campo poco legitimado por la comunidad universitaria: el estudio de la didáctica. El trabajo favorece la concentración de la investigación en el ejercicio de la práctica docente, donde los estudiantes dan a conocer sus sentires, apreciaciones o valoraciones que encuentran en las formas como se desarrolla cada una de las prácticas pedagógicas por parte de los docentes del Departamento de Humanidades. La investigadora halla que los profesores caracterizan y personalizan ciertos estilos de enseñanza que ella denominó "configuraciones didácticas".

La Dra. H. Dámaris Díaz (1999), de la Universidad de los Andes (Venezuela), adelantó una investigación en línea de la didáctica universitaria titulada: Referencia imprescindible para una enseñanza de Calidad. El estudio argumentó que: "merece revisar y asumir al maestro con mayor grado científico y rigurosidad profesional. Invita a revisar el "aula de clase", y desde luego las ciencias que le sirven de marco, como la Didáctica Universitaria, con el propósito central de superar la condición de profesores intuitivos e informadores por la de científicos formadores. Abre la posibilidad de preguntar también por la dimensión humanista de ellos y de lo que procuran inculcar a través de sus 
prácticas pedagógicas sistematizadas" (Díaz, 1999: 50).

En la Universidad Pedagógica Nacional de Colombia [UPN] (2008) el equipo integrado por: Darío Alexánder Zerrate Rubio, Humberto Tovar Pachón, Jaime Arias Obregón, Julio Alberto Noguera Ramírez, María Mercedes Callejas Respeto y James Fernández Córdoba, publicó un trabajo titulado: La didáctica Instrumental, donde se manifiesta que "la didáctica como saber que teoriza sobre la enseñanza, es un tema fundamental en la formación del Licenciado en Música" (Zerrate et al, 2008). Allí se expone que la didáctica debe partir de la enseñanza de los instrumentos musicales. Destacan, a su vez, la educación musical como "piedra angular en el desarrollo integral de las personas" (Zerrate et al, 2008: 78).

En la Pontificia Universidad Javeriana de Bogotá, a través de su programa de Maestría en Educación, se desarrolló en el 2004 la investigación titulada: Las interacciones profesor-estudiante en los procesos formativos de la educación superior del programa de odontología de la Pontificia Universidad Javeriana, desarrollada por la estudiante Francina María Arregocés. Esta investigación además de proponerse caracterizar las interacciones profesor-estudiante, pretende analizar cómo influyen dichas interacciones en los procesos formativos del programa de odontología. Es una investigación que permite fortalecer la idea de que las didácticas, entendidas como "herramientas" que facilitan la interacción entre estudiantes y docentes, favorecen la constitución integral del profesional; la investigación reafirma que los horizontes de sentido emergen e integran mundos de vida en la relación del maestro con el estudiante, puesto que dinamizan y amplían otros mundos posibles en el proceso de construcción del conocimiento. (cf. Arregocés, 2000: 22).
La Universidad Industrial de Santander [UIS] de Bucaramanga, a través de su Maestría en Pedagogía promueve investigaciones que desarrollan profesores y estudiantes, una de ellas titulada: Reflexión sobre la propia práctica en la clase de contabilidad, de Isabel Infante Rojas (2005), busca razones de su propia práctica educativa para generar la mejor perspectiva para la enseñanza y el conocimiento en el área contable. El estudio dejó una enseñanza profunda a los maestros sobre la forma como los estudiantes ven a su profesor en el aula; los miedos, temores y motivaciones que despiertan, según las actividades que se desarrollen en clase. (cfr. Rojas, 2005).

De igual manera, se destaca el proyecto de estudio elaborado por María Consuelo Olaya López (2005) con el título: Las Prácticas Pedagógicas de Inglés: Una exploración de sentido en los estudiantes de educación básica de la Universidad Cooperativa de Colombia, seccional Bucaramanga (UCC). Busca con este proyecto conocer más a fondo la realidad del área de inglés dentro del programa de licenciatura en educación básica con énfasis en humanidades. Otra intención del estudio fue descubrir el significado que tiene para los estudiantes los espacios pedagógicos de contacto con el inglés, de modo que se lograran diseñar estrategias para introducir innovación y ratificar procesos actuales. (cf. Olaya, 2005)

Al indagar sobre los anteriores procesos de investigación que han desarrollado estas instituciones de educación superior, se constata de alguna manera que la investigación sobre la didáctica se ha mostrado paradigmática y a su vez problémica; es decir, vista como un referente universal sobre el que recaen múltiples miradas de estudio, que depende de los contextos y de los saberes sobre los que se busca ver, analizar y proponer nuevas vías por las que se pueda acceder 
no sólo al conocimiento sino a la concreción de actitudes ante la vida.

De las investigaciones halladas, pocas tienen que ver con didáctica, no se hace referencia al ejercicio aplicativo en la práctica educativa de las humanidades. Adicionalmente a dicha exploración investigativa, se deduce una limitada, por no decir, nula investigación sobre horizontes de sentido en su relación con la aplicación de didácticas.

La ausencia de estudios conocidos en la línea de didácticas-horizontes de sentido, se considera razón para expresar que hay una oportunidad valiosa para explorar e investigar en el orden de las didácticas en función de horizontes de sentido, no sólo desde las comunidades educativas sino de las distintas instituciones que proyectan su ser sobre un ejercicio social, como de todo grupo humano que se preocupa por su formación humana.

\section{Desarrollo de la Investigación}

Didáctica y Horizonte de Sentido, se constituyen en objeto de estudio para esta investigación que responde a intereses tanto del departamento de humanidades como de la universidad.

La investigación plantea la siguiente cuestión: ¿Dentro de las prácticas pedagógicas aplicadas por el Departamento de Humanidades, qué didácticas generan horizontes de sentido en los estudiantes de Derecho de la Universidad Santo Tomás, seccional Bucaramanga?

Las características del problema hacen que la investigación se inscriba en lo pedagógico-cualitativo.

Taylor y Bogdan, “definen la metodología cualitativa como aquella que produce datos descriptivos: las propias palabras de las personas; habladas o escritas y la conducta observable" (1986: 30). Para responder a los objetivos del proyecto, es pertinente la metodología cualitativa dado que el estudio se ocupa de una población determinada por docentes y estudiantes de una facultad en particular desde los que mediante observación y entrevistas se deducen, exploran, identifican e interpretan conductas, comportamientos, lenguajes, signos, expresiones, procedimientos y quehaceres, entre otros.

El matiz cualitativo de la investigación implica la acción y desempeño del maestro de humanidades como del estudiante de derecho, desde donde se analizan: el desarrollo de pensamiento, habilidades, construcción de conductas y actitudes de tipo individual y colectivo como la generación de ambientes educativos, expresados a través de lenguajes y códigos, como señala Roberto Hernández Sampieri: "La investigación cualitativa proporciona profundidad a los datos, riqueza interpretativa, contextualización del ambiente o entorno, detalles y experiencias únicas" (2006: 21)

El fundamento epistemológico de la investigación, por consiguiente, busca desarrollarse desde la metodología de la teoría fundamentada (Grounded Theory), en perspectiva teórica al problema planteado, desarrolla un proceso de interpretación de los fenómenos que emergen en las prácticas pedagógicas que desarrollan los docentes del departamento de humanidades en la facultad de derecho, a fin de comprenderlos, al igual que las proposiciones emanadas de la población observada.

La naturaleza del problema en desarrollo, desde el enfoque cualitativo se circunscribe en lo etnográfico, por las razones ya expresadas: abordar a una población educativa en el desarrollo propio de sus prácticas y dinámicas. El método cualitativo y la tradición etnográfica constituyen una mejor ayuda para el entendimiento crítico de las situaciones y fenómenos educativos y por consiguiente para una adecuada y provechosa intervención. 
Según Miguel Martínez... “el enfoque etnográfico se apoya en la convicción de que las tradiciones, roles, normas y valores del ambiente en que se vive se van internalizando poco a poco y generan regularidades que puedan explicar la conducta individual y grupal en forma adecuada". (1997: 28). La etnografía como instrumento en los enfoques cualitativos aporta elementos al presente estudio, como lo señala Carlos A. Sandoval Casilimas, en su obra Investigación Cualitativa, que permite: "la caracterización e interpretación de pautas de socialización, la construcción de valores, el desarrollo y las expresiones de la competencia cultural, el desarrollo y la comprensión de las reglas de interacción, entre otros" (1996: 73).

La investigación focaliza las intencionalidades que mueven los actos (comportamientos dentro de la práctica pedagógica) de la población observada (maestrosestudiantes), el significado que éstos otorgan a sus acciones y las metas que persiguen, en una palabra, la función que desempeñan en el contexto en el que actúan. Se enmarca en el paradigma cualitativo, con matiz etnográfico, que demanda la implementación de herramientas como: la entrevista y la observación, que proporcionan información, tratada mediante la metodología de la teoría fundamentada que, a su vez, implica un ejercicio de triangulación.

Con los anteriores presupuestos se ejecutan y aplican las técnicas de recolección de datos por medio de la observación y la entrevista abierta. El desarrollo de esta fase considera las categorías conceptuales que permiten vislumbrar los horizontes de sentido que reflejan las didácticas.

\section{Instrumentos}

En la investigación cualitativa la observación accede al conocimiento cultural de los grupos, como señala Bonilla (1995) "registra las acciones de las personas en su ambiente cotidiano" (Bonilla y Rodríguez, 1995: 118). La observación fue aplicada en reuniones del Departamento de humanidades y durante el desarrollo de clases de los docentes entrevistados, tenido como referente el problema de la investigación; se aplicó centrada la mirada en aspectos como los actores, lugar, tiempo y comportamientos.

La observación fue no participante con relación a los investigadores ya que se estuvo simplemente como espectadores ajenos a las situaciones; se registran las situaciones a medida que suceden. Los registros de tales observaciones se evidencian en los protocolos de observación.

En el contexto de la investigación, se considera necesario implementar la entrevista abierta. Este instrumento es "útil para indagar un problema y comprenderlo tal como es conceptualizado e interpretado por los sujetos estudiados, sin imponer categorías preconcebidas" (Bonilla y Rodríguez, 1995: 93).

La entrevista final para Docentes y Estudiantes plantea cinco (5) preguntas, que indagan sobre las categorías iniciales (Didácticas aplicadas, Horizontes de sentido y prácticas pedagógicas), que permitieron hallazgos importantes en términos de categorías emergentes y categorías en relación de primer y segundo nivel a partir de las proposiciones allí desarrolladas, fueron posibles estas codificaciones gracias al sistema alfa-numérico que genera Excel.

La investigación adopta la Teoría Fundamentada, presentada inicialmente por Barney Glaser y Anselm Strauss en The Discovery of Grounded Theory (1967) como una metodología que permite el “interjuego" entre la teoría y los datos, de donde emerge nueva teoría. Casilimas (2002) la define como: "una metodología general para desarrollar teoría a partir de datos que son sistemáticamente capturados y analizados; es una forma de pensar 
acerca de los datos y de poderlos conceptualizar" (Casilimas, 2002: 71).

Para Casilimas (2002) “La teoría se va desarrollando durante la investigación en curso mediante el continuo interjuego entre los procesos de análisis y recolección de datos" (2002: 72). Se implementa durante el desarrollo de toda la investigación un ejercicio de análisis que se denomina "análisis comparativo constante" (cf. Glaser and Strauss, 1967); la teoría fundamentada desarrolla una teoría particular denominada "sustantiva" mediante procedimientos de comparación constante de los datos, esta teoría sustantiva se detiene en aspectos muy singulares de la realidad social y seguramente sobre los que no se ha presentado una suficiente comprensión y conceptualización específica.

\section{Participantes}

En el contexto de la investigación, la entrevista abierta se aplica a docentes y a estudiantes, con el ánimo de indagar sobre los objetivos de la investigación. Bonilla y Rodríguez describen el objetivo de la entrevista citando a Patton (1980): "El objetivo de la entrevista cualitativa es conocer la perspectiva y el marco de referencia a partir de las cuales las personas organizan su entorno y orientan su comportamiento" (1995: 93). La entrevista se centra en el conocimiento individual de estudiantes, cuyas edades oscilan entre los 17 y 24 años de edad, de los semestres III, V y VII respectivamente, seleccionados al azar. Estos semestres fueron considerados en cuanto se cree que los estudiantes han adquirido cierto nivel de competencia profesional para identificar problemáticas y proponer salidas a estas.

Los docentes observados, pertenecen al departamento de humanidades y presentan estudios de postgrado y experiencia docente universitaria. Se aplicaron entrevistas que en su orden decantaron preguntas que respondían a cuestiones relativas a las categorías iniciales. Para el caso de docentes y estudiantes el instrumento final se codificó a manera de ejemplo: (E1-03-D2) $=$ (Entrevista 1-Pregunta 3 - Docente 2); para estudiantes: $($ E1-03-E2) $=($ Entrevista $1-$ Pregunta 3 - Estudiante 2).

\section{Análisis de datos}

La teoría fundamentada define un estilo de recolección de datos y análisis teórico, con el ánimo de hacer posible la verificación de las "categorías emergentes" formuladas éstas como proposiciones que plantean relaciones entre conceptos.

Para el análisis de II nivel se definieron relaciones y comentarios, categorías en relación, nuevas proposiciones-citacomentarios, relaciones comentarios.

Siguiendo la secuencia de apartados se compiló la información de docentes (D) y estudiantes (E) por separado, se establecieron las categorías emergentes y en relación en cada uno de sus niveles, para luego proceder a la triangulación como acción final del proceso que otorga validez a los resultados.

La triangulación en la investigación busca cruzar dos informaciones: la primera, relacionada con los referentes teóricos, la segunda, referida a la teoría "sustantiva" que emerge del proceso de la aplicación de la teoría fundamentada.

El cruce de las dos informaciones bajo la acción de la triangulación necesariamente genera una nueva información, para finalmente validar la interpretación y el análisis de la información recopilada en los dos actos citados anteriormente (marco teórico-teoría sustantiva). Validada la información se otorga confiabilidad a los hallazgos de la investigación.

\section{Resultados}

Para establecer una clara relación entre las categorías iniciales referidas: Didác- 
ticas aplicadas, Horizontes de sentido y Prácticas pedagógicas, se consideró importante establecer en primer lugar sobre qué orden de ideas o conceptos docentes y estudiantes las definen. En segundo lugar, se tomaron las definiciones generadas por parte de los entrevistados (docentes y estudiantes) y se procedió a establecer la relación pertinente, respetados los planteamientos de cada uno, a los que se les hizo finalmente un comentario a la luz del marco teórico establecido en la investigación. Vale resaltar que las categorías analizadas responden a los objetivos específicos planteados en la investigación.

\section{Didácticas:}

¿Qué didácticas desarrolla el departamento de humanidades en sus prácticas pedagógicas con estudiantes de derecho?

Para los docentes se infiere que didáctica es una forma o medio (D1-DEDF) que se aplica dentro de un hacer o enseñar en proceso (D2-DCE) en el universo pedagógico (D1-DCE) que, a su vez, provoca la creación de nuevos recursos (D2-DCE).

Los estudiantes manifiestan que didáctica es un ejercicio estratégico (E1-E1-01) que encarna múltiples expresiones posibles de abordar (E1-E1-01) de forma particular, se constituye en recurso para el desarrollo de un método (E1-VRM), este ejercicio particular valora la interacción (E1- EDVI$D E)$ y favorece el trabajo grupal (E1-VRM) mediante los cuales se da a entender o comprender un tema (E1-DEM).

Expuestas las dos percepciones (docentes-estudiantes), para los docentes es particular que la didáctica figure como un "recurso o medio" en su práctica educativa, dado que ante la pregunta: ¿Qué entiende por Didáctica? dan respuestas en las que no hay claridad sobre lo que comprenden por didáctica, lo que dificulta a su vez identificarlas. Los docentes identifican algunas actividades como didácticas, por ejemplo: "interacción (D2VMGC)"; "Análisis vivencial de la Realidad (D1-04D1); “identificación y valoración de casos (D1-VEC); "proyectos individuales (D1-E1-05-AC)"; entre otros.

Sin embargo, el análisis en desarrollo permite comprender que la didáctica aparece dentro de un lenguaje universal en el imaginario de docentes (E1 D1-DCE Didáctica contenida en la enseñanza), en cuanto se constituye en fuerza, presente en la condición humana que se canaliza en la educación, "es el hacer" en el proceso de la enseñanza.

Al analizar la respuesta de los estudiantes, curiosamente, ellos tienen mayor claridad sobre qué es didáctica, respecto a la conceptualización ofrecida por los docentes. Se percibe en el lenguaje del estudiante que la didáctica se orienta al desarrollo de estrategias (E1-E1-01) en aras a la implementación de un método (E1-VRM) a la hora de dar a conocer o impartir un tema, donde desarrolla y aplica sus conocimientos y facultades. No obstante confunden actividades con tipos de didáctica. Se citan algunos ejemplos: "trabajo grupal (E1-VTG)"; "Ver vídeos, hacer ensayos (E1-05-E1)" entre otros.

Dentro del lenguaje del maestro se presenta la didáctica como una capacidad de enseñar (DCE) E1 D2. Sin embargo, dicha conceptualización se reduce a una expresión, que no trasciende teóricamente el ámbito educativo.

Para los docentes es significativo el que la didáctica genere medios (Didáctica Genera Medios: DGM), encargados de dinamizar el proceso de enseñanza, luego la dinámica se manifiesta en el surgimiento de las diversas formas de enseñar (Didáctica es Enseñar en Determinadas Formas: DEDF). A pesar que los docentes tengan claro que la didáctica se manifiesta en diversos tipos de acuerdo a los contextos y población, no logran definir su 
didáctica aplicada al igual que no identifican el patrón de didáctica que sigue el departamento de humanidades. Subyace aquí una tarea por clarificar y puntualizar en miras a la misión del departamento., esto con el ánimo a que el "el desempeño idóneo (del departamento) requiere de la integración de estrategias metacognitivas, cognitivas, afectivo-motivacionales y actuacionales, así como la planeación, monitoreo y evaluación de los mismos, de acuerdo con determinados objetivos.

Las ideas expuestas por docentes y estudiantes en torno a la pregunta por la didáctica ofrecen una oportunidad de reflexión conjunta tendiente a clarificar patrones didácticos afines a su quehacer, favoreciendo así lo trazado por didáctica en el PEl, “(...) significa el arte de enseñar; (...) se refiere a las formas de enseñar, a los diversos componentes de la enseñanza, (...) tendientes a orientar el aprendizaje en forma eficaz (...) la didáctica investiga, experimenta y aplica nuevas técnicas de enseñanza en orden a un aprendizaje eficaz y significativo" (PEI, USTA, 2004:174).

Una didáctica definida para el departamento de humanidades, contribuiría a la unidad entre práctica y misión institucional, dado que estas dos realidades se manifiestan por separado en esta perspectiva de análisis. "al estudiante no le gusta ni le dice nada el discurso abstracto y lejano a su realidad, le interesa lo inmediato y aquello que toque su vida e interés profesional, de lo contrario no trasciende la doxa del maestro frente al discurso vital del estudiante" (Interpretación de la proposición: E1-05-E1-DAPPHS).

Los estudiantes dejan ver que la didáctica está al servicio del método pedagógico como estrategia que otorga valor a la interacción docente-estudiante E1-E1-01 (EDVIDE). Se convierte la didáctica como un recurso para el desarrollo metodológico (VRM). Por ser recurso metodológico favorece el conocimiento personal e igualmente permite favorecer el trabajo grupal (VTG).

En cuanto a la visión de los docentes, manifiestan que la didáctica como fuerza o capacidad para enseñar (D2-DCE) se plantea desde el pensamiento y la experiencia. Lo anterior, establece concordancia con lo que señala (E1- VRM) en sus nociones de didáctica como una estrategia para el desarrollo de un método, que incentiva eficazmente el aprendizaje.

Respondiendo a la pregunta inicial, que interroga por las didácticas que aplica el departamento de humanidades en la Facultad de Derecho, se halla que existen unas prácticas pedagógicas que desarrolla dicho departamento por medio de sus docentes, expresadas en el uso y aplicación de recursos o acciones (estudios de caso, debates, lectura de textos...) que hacen parte de unas didácticas no definidas, por tanto, es necesario indagaR sobre la pretensión intrínseca de dichas actividades, con el fin de determinar sobre qué didácticas se inscriben, para facilitar así el norte de las prácticas pedagógicas en dirección a horizontes de sentido en los estudiantes como en los mismos docentes.

\section{Horizontes de Sentido:}

¿Qué horizontes de sentido generan las didácticas aplicadas por el departamento de humanidades en los estudiantes de derecho?

Considerando los datos ofrecidos por docentes y estudiantes frente a los interrogantes referidos a horizonte de sentido, se tienen los siguientes hallazgos:

Por horizontes de sentido, el docente comprende el ambiente relacional (D2PGS) fundado (D2-VMGC) en los estudiantes, mediante proceso (D1-E1-PSPF), en el que tanto la práctica pedagógica (Docente Dos: Práctica Genera Sentido: D2PGS) como el estudiante (D1-PSPF) y la realidad (D2-EVGP, D3-VAVR), cobran un valor de 
igualdad de condición, que conlleva, de esta manera integral, a que el estudiante sea protagonista de sentido (D1-E1-PSPF), mediante el análisis y compromiso con la realidad (D2-E1-EVGP) para fundar así conocimiento (D2-E1-VMGC) para la vida (D3-E1-VAVR) práctica (D2-E1-PGS).

El estudiante comprende por horizontes de sentido aquellos efectos producidos en ellos por la acción del docente (E3-E1TDEGEE), efectos traducidos en conocimiento (E1-E1-VSC, VETRC), testimonio (E3-E1-TDEGEE), participación activa en solución de problemas (E1-E1- VPASP), en juicio y decisión (E3-E1-HCJD). Dichos efectos son generados por un proceso de práctica pedagógica (E1-VSC), centrada en un trabajo de aula (E1-E1-VTA), en el testimonio por parte del docente (E3-E1TDEGEE) y en el ejercicio experiencial de solución de problemas o conflictos sociales (E1-E1-VPASP, VSPRCS), los cuales aparecen equiparables.

En relación con la definición inferida por los docentes se considera que existe corresponsabilidad en la definición, en tanto que los elementos constitutivos de ambos son semejantes. Se destacan en las definiciones y en orden a su importancia los conceptos de: a) fundar- significado, conocimiento, testimonio; b) proceso de práctica y/ o trabajo de aula; c) realidad y conflictos sociales; d) estudiante; e) docente y f) ambiente relacional.

Para los estudiantes (conocimiento, testimonio y práctica) figuran dentro de un orden más claro en los que evidencian la importancia central desde tres ejes en la generación de horizontes de sentido como son: el trabajo de aula, el testimonio por parte del docente y el ejercicio experiencial. Lo anterior permite guardar coherencia con la afirmación teórica de que "la realidad de la vida cotidiana se me presenta además como un mundo intersubjetivo, un mundo que comparto con otros (...) En realidad, no puedo existir en la vida cotidiana sin interactuar $y$ comunicarme continuamente con otros" (Berger y Luckmann, 2001, 40-41), que constituiría la práctica profesional como tal. Lo que los estudiantes destacan básicamente estaría en orden a los principios o ejes que Delors destaca para el alcance de una verdadera educación y práctica educativa:

aprender a conocer, es decir, adquirir los instrumentos de la comprensión, aprender a hacer, para poder influir sobre propio entorno; aprender a vivir juntos, para participar y cooperar con los demás en todas las actividades humanas; por último, aprender a ser, un proceso fundamental que recoge elementos de los tres anteriores. Estas cuatro convergen en una sola, ya que hay entre ellas múltiples puntos de contacto, coincidencia e intercambio. (Delors, 1996: 96)

En tanto, para los docentes se destaca como eje fundamental el proceso en orden a un ambiente relacional:

el trabajo del docente no consiste tan sólo en transmitir información ni siquiera conocimientos, sino en presentarlos en forma de problemática, situándolos en un contexto y poniendo los problemas en perspectiva, de manera que el alumno pueda establecer el nexo entre su solución y otros interrogantes de mayor alcance. La relación pedagógica trata de lograr el pleno desarrollo de la personalidad del alumno respetando su autonomía... (Delors, 1996: 166).

Incluye igualmente un análisis en el que se hace enfático el aspecto de "proceso" en la versión de los estudiantes, en tanto los docentes confluyen algo indeterminados, es decir demanda mayor esfuerzo para identificar los tres sub-ejes: el docente, el estudiante y la realidad.

En lo correspondiente a estudiantes, llama la atención que no vean al docente 
como una figura relevante en su proceso de formación en cuanto imparte conocimiento, sino en cuanto imparta testimonio de vida, de esta forma se está de acuerdo con Delors:

"significa que esperamos mucho del personal docente..., el aporte de maestros y profesores es esencial para preparar a los jóvenes (profesionales), no sólo para que aborden el provenir con confianza, sino para que ellos mismos lo edifiquen de manera resuelta y responsable" (Delors, 1996: 161).

Es así como la labor del docente, determinaría de alguna manera la iniciativa y sentir del estudiante ante los desafíos que le demanda su realidad, "Cuanto más graves son los obstáculos que debe superar el alumno, pobreza, medio social difícil, incapacidades físicas, más se le exige al maestro. Para obtener buenos resultados, éste debe poder ejercer competencias pedagógicas muy variadas y poseer cualidades humanas, no solo de autoridad, sino también de empatía, paciencia y humildad" (Delors, 1996. Pág.168), lo que vendría a constituirse en testimonio, compromiso y conocimiento en pro de la vida. Se considera que en este ejercicio educativo el estudiante se constituiría en lugar como en algún momento se dijo "hierofánico" es decir en testimonio (D1E1-PSPF, E3-E1-TDEGEE)

"donde lo bello y lo nuevo emerge, donde la historia crea su curso, donde significado y sentido destellan y a su vez se constituyen en alimento de quien anhelosamente estudia, dando así fuerza para seguir el camino de la vida que todo ser humano espera, para hacer menos pesada su carga y menos fatigosa su carrera" (Derrida, 1998:1).

Desde la perspectiva de los estudiantes el concepto de práctica, experiencia social o trabajo, es sobresaliente, lo que llevaría a comprender que la práctica es muy necesaria en el proceso profesional, en esa medida habría un acercamiento al sentido de la vida. De tal forma que la vida en general, profesional y ordinaria, "se organiza alrededor del $<<$ aquí $>>$ de mi cuerpo y el $<<$ ahora $>>$ de mi presente" (Berger y Luckmann, 2001: 39). Debido a su capacidad de trascender el $<<$ aquí y ahora $>>$, el lenguaje tiende puentes entre diferentes zonas dentro de la realidad de la vida cotidiana y las integra en un todo significativo. De esta manera estudiantes y profesores construyen realidad en cuanto la fundan: en este caso el pensamiento del docente exalta el valor de la vida como generador de amplio conocimiento. Cfr. en (D2-E1-VMGC, E1-E1-VSC, VETRC); Ia desarrollan: porque la vida misma del maestro y del estudiante en la medida que se encuentra con el conocimiento da paso a nuevas perspectivas Cfr. (Enseñanza desde la Vida Genera Paradigmas EVGP); la significan: cuando dentro del lenguaje y la experiencia desde la vida y los problemas se constituyen en una práctica que da sentido. Cfr. (PDS); y la hacen conocimiento: al permitir un diálogo y una interrelación de saberes que se convierten en un hacer práctico para dar posibilidad a la significación. Cfr. (Diálogo Interrelacional y Hacer Práctico Fundamento de Significación) (DIHPFS)

Se retoma el principio donde "la vida toda es el fruto de un permanente contacto de la subjetividad con las influencias exteriores que se rechazan, se aceptan o se transforman, pero que nunca están ausentes, sino presentes en forma concreta y real en la educación" (Revista Franciscanum. Revista de las Ciencias del espíritu. No. 21, Enero-Abril, 1999, pp.25).

Finalmente, ya lo decía Comenio citando a Aristóteles, "el hombre de juicio práctico (ético) define su comportamiento aprehendiendo la situación en su singularidad y conflictividad. El proyecto de vida se realiza en condiciones his- 
tóricas concretas. De ahí la centralidad de la categoría de vida, en un sentido ético-cultural" (Rebellato, José Luis. Horizontes éticos en la práctica social del educador).

Precisando algunos elementos esenciales como: a) fundar- significado, conocimiento, testimonio; b) proceso de práctica y/ o trabajo de aula; c) realidad y conflictos sociales; d) estudiante; e) docente y f) ambiente relacional, constituyen no unos "horizontes de sentido de vida" sino ligeros esbozos de significación que se levantan desde la práctica pedagógica. Es innegable que la ausencia de didácticas definidas traiga como consecuencia, a su vez, la ausencia de unos horizontes de sentido de vida claros.

Queda la incertidumbre de saber, si sobre el quehacer educativo de los docentes de humanidades se estén construyendo y promoviendo horizontes de sentido. En el análisis emergen algunos destellos que se ajustarían al conocimiento (E1-E1-VSC, VETRC), al testimonio (E3-E1-TDEGEE), a la participación activa en solución de problemas (E1-E1-VPASP), y a la capacidad de juicio y decisión (E3-E1-HCJD). Los cuales, no es claro si tienen su génesis en las actividades desarrolladas por el docente; por el papel activo que desarrolla la ausencia de unos horizontes de sentido de vida, o por la tarea protagónica del estudiante.

\section{Discusión de resultados}

La discusión se genera a partir del encuentro dialógico entre los datos obtenidos y el análisis generado sobre las categorías: didácticas aplicadas y horizontes de sentido, contenidas en los objetivos de la investigación.

Dentro del ejercicio de análisis se percibe que dichas categorías no existen aisladas de la práctica pedagógica, sino que por el contrario la constituyen, la hacen comprensible y a su vez la explican, por tal razón pretender hablar de didáctica es entrar en el campo de la praxis pedagógica, a su vez, pretender hablar de pedagogía es "habérnoslas" con la didáctica. Por otra parte, y sin duda, no es desconocido para el mundo de la educación que la pedagogía incluye una fuerza intencional que induce tanto al educando, al educador como a la institución a articular los ánimos, las estrategias y los contenidos a la adquisición, generación y desenlace de logros que, por lo general, desembocan en el ejercicio de la vida.

Por lo anterior, la discusión condensa las miradas sobre didácticas aplicadas y horizontes de sentido fusionadas en las Prácticas Pedagógicas.

Se consideraron las Prácticas Pedagógicas (PP) como el conjunto de estrategias (E1-E1-01, D1-DEDF) e instrumentos que utiliza el profesor "universitario" en el desarrollo de sus clases, con la pretensión de formar a los estudiantes en el marco de la excelencia académica y humana. Además se advirtió que en su dimensión estratégica involucran cuatro elementos activos e interactivos como son: estudiantes - maestros (D1-E1-PSPF), saberes pedagógicos y la misma Universidad, que permite asumirlas como escenarios dinámicos y dialógicos; facilitan la generación de conocimiento (D2-E1-VMGC, E1-E1-VSC, VETRC) de forma colectiva y procuran el enriquecimiento del saber pedagógico y su proyección edificante en la cultura.

¿Qué prácticas docentes permiten construir significación y sentido?

Al relacionar las diversas respuestas, el docente (D1) en concordancia con (D3) coincide en afirmar que la práctica pedagógica mantiene una relación definitiva con la enseñanza, trasciende los contenidos y centra su atención en la vida misma (VRAV), la cual se constituye en insumo de trabajo u objeto de estudio. Estas miradas están en sintonía 
de igual manera con las afirmaciones de Delors:

el trabajo del docente no consiste tan sólo en transmitir información ni siquiera conocimientos, sino en presentarlos en forma de problemática, situándolos en un contexto y poniendo los problemas en perspectiva, de manera que el alumno pueda establecer el nexo entre su solución y otros interrogantes de mayor alcance. La relación pedagógica trata de lograr el pleno desarrollo de la personalidad del alumno respetando su autonomía... (Delors, 1996: 166)

Por su parte los estudiantes hallan sentido a la práctica pedagógica cuando hay diálogo con el maestro: para (E1-E1-02) la práctica pedagógica se da en términos de "concertación de trabajo entre docenteestudiante" (VCTDE), mientras que en (E1-E2-02) la relación docente-estudiante se evidencia en un ejercicio de enseñanza sustentado en la actitud crítica (MEBAC), el cual demanda un proceso soportado en argumentos (SRPCCA), que para (E3) desemboca en la consolidación profesional intencional (PPECPI).

Nótese aquí cómo el estudiante da especial importancia a la comunicación constante con el docente, la cual le permite establecer acuerdos en el desarrollo del ejercicio pedagógico, es toda una vivencia dialógica, la que en su imaginario define la práctica pedagógica; en este sentido se relaciona la postura de (D2) para quien la enseñanza es un ejercicio práctico de diálogo y escucha sobre los cuales se forma la capacidad de interiorizar (EPCI), que se hace efectiva mediante la identificación y valoración de casos (D1-VEC) a través de una estructura que se constituye en la práctica de la interpretación, el análisis, la argumentación y la proposición, por medio de las cuales el maestro "jalona el conocimiento" (IAPJC) e interrelaciona saberes y sentires (D3 -EISS).
Montero (1987) sustenta esta dimensión dialógica en las prácticas pedagógicas al afirmar que las prácticas pedagógicas se entienden como las experiencias de comunicación e interacción humana, las cuales permiten comprender el tipo de formación que promueve la Institución Educativa, dan cuenta del sistema evaluativo imperante, de las formas de comunicación entre los agentes educativos, del tipo de enseñanza-aprendizaje que promueven los docentes y de la forma como éstos utilizan los recursos didácticos en el proceso. (Montero, 1987)

Un valor agregado a la comunicación en las prácticas pedagógicas es el conocimiento cuyo dominio, en últimas permite la generación de didácticas y horizontes de sentido, por ello para (E3) la práctica pedagógica es una labor donde el dominio de conocimiento, favorece la creatividad y la comunicación (DEDCC); en (E1), el conocimiento adquiere valor, en la medida que se hace trasversal e interdisciplinar (TCTI), para (E2 y E1) la práctica se constituye en generadora de sentido (PDS), de significación y fuente de perspectivas (SGP, VSSPP).

En la pretensión de interpretar las didácticas que aplica el Departamento de Humanidades en sus prácticas pedagógicas e identificar los horizontes de sentido que generan tales didácticas en las prácticas pedagógicas, es claro que tanto para docentes y estudiantes son bien significativas tales prácticas pedagógicas, llámense clases, salidas de campo, eventos del departamento y toda acción pedagógica que implique la presencia del departamento, en tanto generan conocimiento, integran al estudiante y procuran canales de diálogo abierto en los participantes; nótese que no de desliga práctica pedagógica de la enseñanza, práctica pedagógica del conocimiento y práctica pedagógica de la experiencia dialógica. 
Retomando la pregunta: ¿Qué prácticas docentes permiten construir significación y sentido? es posible decir que todas aquellas prácticas que evidencien en el maestro conocimiento pero que, a su vez, generen conocimiento; aquellas prácticas donde el diálogo y el consenso son baluarte esencial y todas aquellas que transversalicen el conocimiento y se nutran de otros saberes, serán aquellas prácticas que dejen ver una didáctica particular y generen sentido en los estudiantes.

Hecho el análisis de la información, direccionado por las categorías, se hace notoria la importancia de las prácticas pedagógicas en el ámbito del docente y el estudiante, pues desarrolla principios como: el encuentro de saberes entre docente y estudiante, la posibilidad de diálogo y un conocimiento que atrae y lleva a un compromiso testimoniado. Estos tres elementos permiten ver un horizonte de sentido que tiene su origen básicamente en las prácticas pedagógicas.

\section{CONCLUSIONES}

El ideario educativo de la Universidad Santo Tomás, promueve la formación integral de las personas, en el campo de la Educación Superior, mediante acciones y procesos de enseñanza-aprendizaje. A este propósito misional, se suman las prácticas pedagógicas ejecutadas por los docentes del Departamento de Humanidades de Bucaramanga, referente inspirador de la investigación, que permitió a su vez ejecutar un interesante trabajo de reflexión educativa en torno a la pregunta: ¿Dentro de las prácticas pedagógicas aplicadas por el Departamento de Humanidades, qué didácticas generan horizontes de sentido en los estudiantes de derecho de la Universidad Santo Tomás de Bucaramanga?

La investigación permitió un amplio espectro de conceptualización y revisión documental que contribuyó a la evolución del empeño trazado; abordar las prácticas pedagógicas, didácticas y horizontes de sentido en el contexto institucional-local, pero también desde la reflexión teórica general, exigió un interesante trabajo, no sólo por su abordaje, sino a la hora de hacer lectura interpretativa de los hallazgos en relación con lo ya definido por teóricos y documentos institucionales.

El trabajo investigativo, permitió un acercamiento cuidadoso a la labor pedagógica del Departamento de Humanidades en la facultad de Derecho, desde donde se consideró la información obtenida a través de los estudiantes colaboradores; a su vez, los docentes del departamento, fueron fuentes fundamentales a la hora de implementar el estudio, y aportaron desde su saber y hacer pedagógico.

La misma pregunta problémica de la investigación, permitió establecer una categorización que conllevó a estructurar bajo una matriz operativa de objetivos y otra de coherencia metodológica, dichas categorías, con el ánimo de profundizarlas, indagarlas y relacionarlas sistemáticamente.

Se hizo necesario acudir a la reflexión pedagógica - tomista en torno a las disertaciones sobre educación y formación, no sólo como eje doctrinal sino como principio inspirador y referente propositivo, tanto para docentes como para estudiantes.

Por otra parte, la ausencia de didácticas definidas, hace más complejo identificar los horizontes de sentido que de ellas podrían generarse en el proceso pedagógico. No obstante, los docentes comprenden que tales horizontes pueden forjarse en el ambiente relacional -encuentro- fundado en los estudiantes, mediante procesos, en los que, tanto la práctica pedagógica como el estudiante y la realidad, cobran un valor de igualdad de condición, conllevan de esta manera a que el estudiante sea protagonista de sentido, mediante el análisis y compromiso 
con la realidad y generan conocimiento para la vida práctica.

Para el estudiante es importante el referente que constituye el maestro, visto como un modelo inspirador y posibilitador de conocimiento; su testimonio enseña y lanza al discente a integrar activamente procesos de: participación, discusión y búsqueda de soluciones a problemáticas del orden personal y social. Se resalta esta postura, ya que el maestro enseña desde el ejemplo, y el estudiante al hacer lectura de lo que ve y valida en su modelo (docente) configura sentidos y compromisos para su propio ser y hacer. El estudiante-de derecho- tiene en cuenta y toma para sí el discurso docente, pero lo apropia entonces, cuando descubre en su maestro una coherencia entre su discurso y praxis, es aquí precisamente cuando consolida su propio horizonte de sentido de vida. Llama la atención cómo el testimonio es un referente clave en la construcción de horizonte de sentido, seguramente un elemento que pasa desapercibido en la práctica pedagógica de los docentes pero que el estudiante como agente activo recupera y observa para sí, este es su mejor discurso cuando de construir norte y camino en la vida del estudiante se trata.

Lo anterior suscitó la interrogación del grupo investigador, sobre el quién construye horizonte de sentido de vida: -el maestro en el estudiante a través de su actividad (didáctica) pedagógica o -el mismo estudiante desde la lectura que hace de tales actividades con estrategias particulares. Es posible responder -con Gadamer- se genera una "fusión de Horizontes", donde el maestro movido por su horizonte de vida, lo comunica al estudiante, quien a su vez encarna un horizonte personal de vida. Estos horizontes (estudiante y docente) confluyen en cierto modo en el encuentro y desarrollo de una actividad pedagógica; de ahí el interrogante que ahora resulta: ¿Quién construye el horizonte?
La pregunta anterior lleva a plantear el problema de investigación de manera inversa, dada la dificultad hallada en la "fusión de horizontes" es decir: ¿No serán los horizontes de sentido presentes en maestros y estudiantes, los que demandan la generación de didácticas a través de las prácticas pedagógicas?

En relación a Prácticas Pedagógicas, se concluye que no son referidas ni por maestros ni estudiantes más allá del aula, se habla de ellas a partir del trabajo que se desarrolla en el salón de clases sin trascenderlo, aunque muchas actividades impliquen un trabajo de indagación externa, matizada por lecturas, análisis de realidades o casos, no se otorga fuerza a este trabajo extramural en el que el docente se vea implicado directamente.

Para los estudiantes es valiosa la práctica que contenga dentro de sí estructuras de interpretación, manifestadas en análisis, argumentación y proposición, por medio de las cuales el maestro facilitaría "jalonar el conocimiento" e interrelacionar saberes y sentires. Son este tipo de prácticas, las que despiertan mayor interés en la vida del estudiante.

El estudio afianzó el ejercicio de la investigación como valor agregado de la Maestría en Educación, ya que se investigó la misma práctica pedagógica y la labor formativa del departamento de humanidades en el contexto de la educación superior. Se comprendió el valor de seguir disciplinadamente una estructura que daba norte y orden a medida que se desarrollaba la investigación. Es importante resaltar el valor que tuvo durante el desarrollo del estudio la triangulación, como ejercicio que permitió el encuentro de datos emergentes que con la ayuda de la teoría fundamentada se contrastaron durante todo el proceso, hasta consolidar los hallazgos registrados.

La estructuración del marco teórico del estudio fue una posibilidad importante 
para contrastar la teoría con el ser y quehacer de docentes, estudiantes y del mismo Departamento de Humanidades. Desde aquí se propiciaron jornadas de discusión al interior del grupo investigador en torno a las categorías constitutivas del proyecto.

La investigación posibilitó una mirada introspectiva al Departamento de Humanidades, dio cumplimiento a la revisión constante de sus prácticas pedagógicas -en la facultad de derecho-, la cual abre nuevas perspectivas de investigación para otras facultades; como también nuevos desafíos al Departamento de Humanidades y a la universidad en Bucaramanga.

Finalmente, permitió reflexionar en torno a un fenómeno educativo: las didácticas, en cuanto son interpretadas, asumidas y desarrollas de diversas maneras según los contextos, donde incide de manera categórica la formación del docente y la comprensión particular que se tiene de la filosofía de la institución, que marcan la discusión y comprensión del tema.

\section{Referencias}

ARREGOCÉS, F. (2004). Las interacciones profesorestudiante en los procesos formativos de la educación superior del programa de odontología. Pontificia Universidad Javeriana. Bogotá, Barcelona. Pág. 22.

BARNEY GLASER Y ANSELM STRAUSS (1967). The discovery of grounded theory.

BERGER L. Y LUCKMAN T. (2001). La Construcción Social de la Realidad. Buenos Aires, Amorrortu.

BONILLA, E. (1997), Más allá del dilema de los Métodos. Édiciones Norma. Bogotá

DELORS, J. (1996.) La educación encierra un tesoro. Editorial: Santillana, Ediciones Unesco. Madrid

DÍAZ, D. (1999). La Didáctica universitaria: referencia imprescindible para una enseñanza de calidad. Universidad de los Andes, Venezuela. Pág. 1-10.

GÓMEZ, R. (1999). Revista de las Ciencias del Espíritu. Franciscanum. Vol. 4

HERNÁNDEZ, R. (2001). Metodología de la Investigación. Editorial: McGraw-Hill, 2 Edición, México.

INFANTE, R. (2005). Reflexión sobre la propia práctica en la clase de contabilidad. Universidad Cooperativa de Colombia.

JARABO, F, (2003). Las tecnologías de la información y las comunicaciones. Universidad de la Laguna, Salamanca: España.

MARTÍNEZ, M. (1997). La investigación cualitativaetnográfica en educación. Universidad Simón Bolívar. Venezuela.
MONTERO, L. (1987) Las prácticas de enseñanza en la formación de los profesores: sentido curricular y profesional. Santiago de Chile: Actos Symposium Noción sobre Prácticas de Enseñanza. Pág. 38

OLAYA L. (2005). Las Prácticas Pedagógicas de Inglés. Universidad Cooperativa de Colombia, seccional Bucaramanga.

REBELLATO, J. (1997). Horizontes éticos en la práctica social del educador. Centro de formación y estudios del INAME, Montevideo.

RIVAS, C. (1999). Investigaciones sobre el sentido. Editorial: Argos.

SANDOVAL, C. (1996). Investigación Cualitativa. Módulos de investigación Social, ICFES.

TAYLOR Y BOGDAN. (1986). Introducción a los métodos cualitativos de investigación. Editorial: Paidós, Barcelona.

TOBÓN, Sergio, (2004) Formación Basada en Competencias. Kimpres.

USTA, (2002) Proyecto Educativo Institucional. (PEI).

ZERRATE, (2008). La didáctica Instrumental. Universidad Pedagógica Nacional [UPN]. Bogotá. 